\title{
Tratamiento del síndrome de apneas e hipopneas obstructivas del sueño con terapia miofuncional orofaríngea: Experiencia en hospital público de Chile
}

\author{
Treatment of obstructive sleep apnea syndrome with \\ myofunctional oropharyngeal therapy: Experience in public Hospital of Chile
}

Mario Díaz S', Ana Salazar C', Felipe Bravo G'1, Adrián Ocampo-Garcés².

\begin{abstract}
RESUMEN
Introducción: El síndrome de apneas e hipopneas obstructivas del sueño (SAHOS) afecta al 2\%-4\% de las personas adultas. EI CPAP es la principal terapia en casos moderados y severos, pero sólo es tolerado en el 50\%-70\% de los pacientes. La terapia miofuncional orofaríngea (TMO) ayuda a reducir el colapso de la vía aérea superior mediante ejercicios de fortalecimiento de la musculatura orofaríngea. En la última década ha demostrado una buena eficacia en grupos variados de pacientes con SAHOS.

Objetivo: Estudiar efectividad de la TMO en pacientes con SAHOS.

Material y método: Revisamos retrospectivamente 12 pacientes con SAHOS leve y moderado tratados con TMO. Los datos demográficos y polisomnográficos se analizaron antes y después de la terapia.

Resultados: Edad media: $65 \pm 9,0$ años, el 58,3\% eran mujeres, el 33,3\% eran obesos. Observamos una disminución significativa del IAH (13,64 $\pm 1,99$ vs 10,13 $\pm 2,09, p$ =0,008); una mejoría en la eficiencia del sueño, los porcentajes de etapas N3-REM y del índice de microdespertares. También observamos una reducción clínicamente significativa en las puntuaciones de la escala de somnolencia de Epworth, del Mallampatti y el perímetro cervical.

Conclusión: La TMO fue eficaz en la mayoría de los pacientes con SAHOS leve y moderado. Cada unidad de sueño en centros de salud públicos en Chile debe considerar este tratamiento.

Palabras clave: Ejercicios miofuncionales orofaríngeos, terapia miofuncional orofaríngea, síndrome de apneas e hipopneas obstructivas del sueño, apneas del sueño, roncopatía.
\end{abstract}

\section{ABSTRACT}

Introduction: The obstructive sleep apnea syndrome (OSA) affects about 2\%-4\% of adults. CPAP is the first indication to treat moderate and severe cases, however

\footnotetext{
1 Complejo Hospitalario San José, Servicio Salud Metropolitano Norte. Santiago, Chile.

2 Programa de Fisiología y Biofísica, Instituto de Ciencias Biomédicas, Facultad de Medicina. Universidad de Chile, Santiago, Chile.

Los autores declaran no tener conflictos de interés.
}

Recibido el 8 de abril, 2019. Aceptado el 12 de julio, 2019. 
the treatment is tolerated in only $50 \%-70 \%$ of patients. Therapy with myofunctional oropharyngeal (TMO) exercises helps to reduce upper airway collapsibility by strengthening the oropharyngeal musculature, and in the last decades had demonstrated good efficacy in variated groups of OSA patients.

Aim: To study TMO effectivity in OSA patients.

Material and method: We reviewed retrospectively the clinical records of 12 (7 female) mild and moderate unselected OSA patients. Clinical and polysomnographic data were analyzed before and after TMO.

Results: The mean age of patients was $65.0 \pm 9.0$ years and median BMI was 26.7 $\mathrm{kg} / \mathrm{m} 2$. TMO was associated to a significant reduction in median of Epworth somnolence scale (11.0 vs. 7.0), median apnea hypopnea index (13.4 vs 9.0 events/h), and decrease in cervical circumference. There was a tendency to improve quality of NREM sleep with increases in N3 stage and decrease in arousal index.

Conclusion: In a real clinical context, TMO reduced the severity of OSA in $41.6 \%$ in the studied patients. Because of its safety and low cost, TMO should be introduced as a therapeutic option in public sleep units in Chile.

Key words: Obstructive sleep apnea, myofunctional oropharingeal therapy.

\section{INTRODUCCIÓN}

El síndrome de apneas e hipopneas obstructivas el sueño (SAHOS) se define en la población adulta como la obstrucción parcial o completa de la vía aérea superior (VAS) durante el sueño, con más de 5 eventos respiratorios/hora y la presencia de síntomas secundarios (ronquidos, pausas respiratorias en el sueño observadas por un cercano, somnolencia diurna excesiva, trastornos cognitivos, etc.). Según datos extrapolados de la Encuesta Nacional de Salud del año 20101', el 4,7\% de los adultos y $7,6 \%$ de los adultos mayores tienen síntomas sugerentes de este trastorno. Los principales riesgos para la salud asociados con el SAHOS son: aumento en la tasa de enfermedades cardiovasculares (hipertensión arterial crónica, arritmia por fibrilación auricular, enfermedad coronaria, enfermedad cerebrovascular), aumento en la tasa de accidentes de tránsito, mayor prevalencia de problemas psicosociales. Además, la presencia de SAHOS se asocia a una peor evolución en otras patologías de base, especialmente enfermedad renal crónica, diabetes mellitus, enfermedad pulmonar obstructiva crónica.

En 1981 Sullivan y cols, publicaron la primera experiencia en la que se demostró la efectividad del CPAP para el tratamiento del SAHOS. Desde entonces el dispositivo sigue siendo la primera línea de tratamiento para este trastorno en casos moderados y severos ${ }^{2}$. Pese a que se han introducido componentes tecnológicos en los equipos que permiten mejorar la adaptabilidad del paciente (termohumidificadores, alivio de presión espiratoria, autoCPAP, etc.) las tasas de adherencia a largo plazo fluctúan sólo en torno al $50 \%-70 \%{ }^{3}$. Dentro de los pacientes con mayor probabilidad de abandonar el tratamiento están los fumadores, los que tienen enfermedades cardiovasculares previas y aquellos con un índice de apneas e hipopneas del sueño (IAH) más bajo4.

Las terapias alternativas al CPAP incluyen los dispositivos de avance mandibular (DAM) y el tratamiento quirúrgico. Sin embargo, ambas terapias tienen baja accesibilidad en nuestro medio, su efectividad terapéutica es variable y no están exentas de efectos adversos ${ }^{5,6}$. En las últimas décadas ha tenido un creciente desarrollo en el tratamiento del SAHOS la terapia miofuncional orofaríngea (TMO), realizada por fonoaudiólogos con entrenamiento en trastornos del sueño. Esta terapia intenta disminuir la colapsabilidad de la VAS a través de ejercicios funcionales repetitivos de la musculatura faríngea. La TMO ha demostrado ser eficaz en reducir la intensidad de los ronquidos y disminuir el índice de eventos respiratorios durante el sueño? 


\section{OBJETIVO}

Con el objetivo de evaluar la eficacia terapéutica de la TMO en un contexto clínico real, realizamos una revisión retrospectiva de las fichas de atención de aquellos pacientes de nuestro hospital que fueron derivados a TMO. En cada paciente se evaluaron parámetros clínicos y polisomnográficos, antes y después del tratamiento con ejercicios miofuncionales.

\section{MATERIAL Y MÉTODO}

Pacientes: Se revisaron retrospectivamente las fichas de pacientes que fueron derivados para TMO entre el año 2016 y 2017. La obtención de datos se realizó entre marzo y junio de 2018. Los pacientes fueron derivados a la Unidad de Trastornos del Sueño por síntomas sugerentes de SAHOS. La primera atención la realizó un neurólogo 0 neumólogo quien solicitó una polisomnografía (PSG). Según la severidad del IAH obtenida en este examen los pacientes fueron derivados para tratamiento con CPAP o TMO con fonoaudiólogo. Una vez finalizada ésta se solicitó una segunda PSG de control. El presente estudio está de acuerdo con los principios de la declaración de Helsinski de la Asociación Médica Mundial (1975) para investigaciones en humanos. Los pacientes fueron incluidos en el estudio de acuerdo a los siguientes criterios: (i) edad mayor a 18 años, (ii) diagnóstico de SAHOS leve (IAH entre 5 y 14,9 eventos/hora), (iii) pacientes con SAHOS moderado (IAH entre 15 y 29,9 eventos/hora) que no toleraron el CPAP y (iv) cada paciente debía contar con una segunda PSG como examen de control posterior a la TMO. Se excluyeron pacientes: (i) que no completaron 0 no asistieron a las sesiones programadas con el terapeuta, (ii) con datos insuficientes en sus fichas clínicas, (iii) o que no eran capaces de seguir instrucciones del fonoaudiólogo. Por tratarse de una población no seleccionada de pacientes, no hubo exclusión por patologías crónicas de base.

\section{Terapia miofuncional orofaríngea}

Se define como un conjunto de técnicas y procedimientos que incluyen el uso de ejercicios isotónicos e isométricos en músculos orofaciales y orofaríngeos, destinados a modificar sus patrones anatómicos y funcionales. Los ejercicios están dirigidos a tratar de mejorar las funciones de respiración, masticación, deglución y habla. La TMO en los pacientes con trastornos respiratorios del sueño está enfocada principalmente en fortalecer la musculatura dilatadora de la faringe para reducir su colapsabilidad ${ }^{8}$. En cada paciente se programó un total 8 sesiones (1 ó 2 veces semanales) y se le indicaron ejercicios a realizar el resto de los días en domicilio durante 5-10 minutos, 3 veces al día, frente a un espejo. Atendiendo las necesidades individuales de algunos pacientes, se realizaron hasta 4 sesiones extra a las programadas. En Tabla 1 se detallan los ejercicios incluidos en la TMO.

\section{Polisomnografía}

Cada uno de los pacientes se realizó un estudio polisomnográfico nocturno (Nicolet ${ }^{\mathrm{MR}}$ ) de acuerdo a recomendaciones de la American Academy of Sleep Medicine (AASM) ${ }^{9}$. Se utilizaron electrodos para electroencefalograma (F3, F4, C3, C4, 01, 02) referidos a mastoides (M1, M2); electrooculograma, electromiografía de superficie en músculos mentalis y tibiales anteriores, estudio de flujo respiratorio con cánula de presión (PTAF) y termistor; oxímetro de pulso, bandas de esfuerzo respiratorio torácica y abdominal. No se contó con sensor de posición corporal ni de ronquidos. El análisis del examen lo realizan neurólogos con formación en medicina del sueño y neurofisiología clínica. La polisomnografía de control fue realizada entre 3 y 6 meses después de finalizada la totalidad de las sesiones de TMO, dependiendo de la disponibilidad de cupos.

\section{Análisis estadístico}

Se analizan las siguientes "variables polisomnográficas" de acuerdo a las recomendaciones de la AASM $^{10}$ : Tiempo total de sueño (TTS, cantidad de sueño en minutos obtenida durante el registro de la polisomnografía). Eficiencia del sueño (porcentaje obtenido al dividir el TTS por el tiempo total en cama). Latencia al inicio del sueño (tiempo en minutos transcurrido desde que se apagan las luces hasta la primera época en etapa N1). 
Tabla 1. Descripción de ejercicios miofuncionales realizados a pacientes con SAHOS leve-moderado

\begin{tabular}{|c|c|c|}
\hline GRUPO MUSCULAR & NOMBRE DEL EJERCICIO & DESCRIPCIÓN \\
\hline \multirow[t]{4}{*}{ MÚSCULOS LINGUALES } & Barrido lingual & $\begin{array}{l}\text { Posicionar punta de lengua en la papila incisiva y realizar } \neq \text { desliza- } \\
\text { miento antero-posterior contra el paladar duro en dirección hacia } \\
\text { el paladar blando. La realización de este ejercicio debe hacerse con } \\
\text { labios cerrados sin contacto dentario ( } 20 \text { veces, } 3 \text { veces al día) }\end{array}$ \\
\hline & Adosamiento linguo-palatino & $\begin{array}{l}\text { Inicialmente debe realizarse chasquidos linguales con contracción } \\
\text { isotónica y luego adosar la lengua contra paladar duro y mantener } \\
\text { el acoplamiento en ejercicio isométrico durante } 5 \text { segundos y lue- } \\
\text { go soltar. ( } 20 \text { veces, } 3 \text { veces al día) }\end{array}$ \\
\hline & Presión lingual contra paladar duro & $\begin{array}{l}\text { Empujar el tercio anterior de la lengua contra el paladar duro, man- } \\
\text { teniendo el sello labial sin contacto dentario, durante } 5 \text { segundos y } \\
\text { luego soltar. ( } 20 \text { veces, } 3 \text { veces al día) }\end{array}$ \\
\hline & Descenso dorso lingual & $\begin{array}{l}\text { Puede ser utilizado "baja lengua" para ayudar el descenso del dor- } \\
\text { so lingual con pequeños toques (desencadenamiento reflejo nau- } \\
\text { seoso). Luego de la contracción voluntaria iniciar el descenso con } \\
\text { contracción isotónica y mantener abajo con contracción isométrica } \\
\text { durante } 5 \text { segundos y luego relajar ( } 20 \text { veces, } 3 \text { veces al día). }\end{array}$ \\
\hline \multicolumn{3}{|l|}{ BUCCINADOR } \\
\hline ORBICULAR DE LOS LABIOS & Dedo en mejilla & $\begin{array}{l}\text { Posicionar dedo en mucosa interna de la mejilla y hacer presión } \\
\text { con el dedo empujando hacia los dientes, realizando contracciones } \\
\text { isotónicas, } 2 \text { veces por lado. Posteriormente las contracciones se- } \\
\text { rán isométricas durante } 10 \text { segundos (10 veces por lado, } 3 \text { veces } \\
\text { al día). }\end{array}$ \\
\hline VELO PALATINO & Vocalización & $\begin{array}{l}\text { Emitir la vocal A de forma intermitente, } 10 \text { veces al día durante } 2 \\
\text { semanas hasta lograr la contracción voluntaria. Luego se retira la } \\
\text { vocalización y se solicita contracción voluntaria de modo intermi- } \\
\text { tente durante } 2 \text { semanas. Luego de obtener la coordinación del } \\
\text { movimiento se solicita contracción de la musculatura mantenida } \\
\text { por } 5 \text { segundos y luego soltar (Realizar } 20 \text { veces, } 3 \text { veces al día). }\end{array}$ \\
\hline
\end{tabular}

Latencia al sueño REM (tiempo en minutos desde el inicio del sueño a la primera época en estado de sueño REM). Porcentaje de etapa N3 (porcentaje obtenido al dividir el tiempo total en etapa N3 por el TTS). Porcentaje de etapa REM (porcentaje obtenido al dividir el tiempo total en etapa REM por el TTS). Índice de microdespertares (número de activaciones transitorias del trazado EEG de 3 a 15 segundos de duración por cada hora de sueño). Índice de apneas-hipopneas (número total de hipopneas, apneas obstructivas, apneas mixtas y apneas centrales por cada hora de sueño). Índice de desaturaciones de oxígeno (número total de caídas de al menos $3 \%$ en la $\mathrm{SaO}_{2}$ dividido por cada hora de sueño). Saturación de $0_{2}$ mínima (valor de saturación de oxígeno mínima observada durante el sueño). Porcentaje del tiempo de sueño con saturación de $0_{2}$ menor a $90 \%$ ("CT 90").

Las "variables clínicas" estudiadas fueron: puntaje en escala de somnolencia de Epworth (consiste en una encuesta de 8 preguntas con puntaje de 0 a 24, que considera el diagnóstico subjetivo de somnolencia diurna excesiva con más de 10 puntos); perímetro cervical (en centímetros, medido bajo prominencia laríngea en paciente sentado); índice de Mallampatti (corresponde a la categorización en cuatro niveles de la cavidad orofaríngea estimada visualmente con boca abierta 
y sin fonación). Se utilizó el test de comparación pareada no paramétrico (test de Wilcoxon) para analizar cada una de las variables clínicas y polisomnográficas obtenidas antes y después de completar las sesiones TMO. Los valores se presentan como mediana de la muestra y su rango intercuartil (RIC). Se consideró para significancia estadística un $\mathrm{p}<0,05$.

\section{RESULTADOS}

\section{Características demográficas de los pacientes}

De un total de 74 pacientes que fueron referidos para realizarse TM0, $15(20,2 \%)$ no completaron 0 no asistieron a las sesiones programadas con el terapeuta o tenían datos insuficientes en sus fichas clínicas; 46 (62,2\%) no contaban con una PSG de control, siendo removidos del estudio. De los restantes trece pacientes que cumplieron con los criterios de inclusión, una paciente fue desestimada del estudio por tener un muy bajo tiempo total de sueño en la primera PSG y por realizarse titulación con CPAP a mitad de noche en el segundo examen.

Los 12 pacientes que fueron incluidos en el estudio $(16,2 \%$ del total de referidos a TM0) tenían una edad promedio de $65 \pm 9,03$ años (52 a 82 años). La distribución según sexo fue de 7 mujeres $(58,3 \%)$ y 5 hombres $(41,7 \%)$. No hubo diferencias significativas de edad entre ambos $\operatorname{sexos}(66,8 \pm 7,4$ vs $63,7 \pm 10,4$ años). Debido a que no existió una selección previa, los pacientes contaban con antecedentes mórbidos relevantes: 7 pacientes con hipertensión arterial crónica, 1 paciente con diabetes mellitus tipo II, 2 con enfermedad de Parkinson de menos de 3 años de evolución, 1 paciente con asma bronquial, 1 paciente con hipotiroidismo. Todos recibían tratamiento para cada una de sus enfermedades.

\section{Variables clínicas}

La mayoría de los pacientes evaluados (10/12) se quejaba de somnolencia diurna excesiva, la mediana en la escala de somnolencia de Epworth (ESS) fue de 11,0 puntos (RIC: 7,0-18). Después de completar las sesiones de TMO se observó una reducción estadísticamente significativa en el puntaje de esta escala, con una mediana de 7,0 puntos (RIC: $3,0-10,0$ puntos, $p=0,023$ ). Después de la TMO 10/12 pacientes redujeron el puntaje de la ESS y finalmente sólo 3 tenían un puntaje $\geq 10$ puntos. La mediana del índice de masa corporal (IMC) en el grupo de pacientes fue de $26,7 \mathrm{~kg} / \mathrm{m}^{2}$ (RIC: 25,6-30,9). Dos pacientes tenían un IMC normal (16,7\%), 6 tenían un IMC en rango de sobrepeso (50\%) y 4 un IMC en rango de obesidad $(33,3 \%)$. Se observó un alza no significativa en el IMC después de la TM0 (28,3 kg/m2 [RIC: $26,2-$ $31,4], p=0,624)$. La mediana del perímetro cervical fue $37,0 \mathrm{~cm}$ (RIC: $37,0-40,5)$. Tres pacientes tenían un perímetro $\geq 40 \mathrm{~cm}$, valor que se asocia a un mayor riesgo de SAHOS. Esta variable también mostró una reducción significativa después de la TM0 (mediana 37,0 cm [RIC: 34,5-39,0], p $=0,017)$. Seis pacientes $(50 \%)$ redujeron el valor de su perímetro cervical después de la terapia.

\section{Variables polisomnográficas}

La PSG basal mostró una media de IAH de 13,4 eventos/hora de sueño (RIC: 7,6-16,2) con un valor mínimo y máximo de 5,1 y 28,6 eventos/hora respectivamente. Ocho pacientes (66,7\%) estaban en rango de SAHOS leve, 4 pacientes $(33,3 \%)$ en rango de SAHOS moderado. EI IAH se redujo significativamente después de la TMO, alcanzado en la PSG de control una mediana de 9,0 evento/hora (RIC: $5,5-11,7 ; p=0,025)$; con 7 pacientes en rango leve (58,3\%), 2 en rango moderado (16,7\%) y 3 en rango normal (25,0\%). 10/12 pacientes redujeron su IAH después de la TMO, la mitad de ellos pasó a un rango de menor severidad del SAHOS.

No se observaron cambios significativos en los parámetros de la oximetría de pulso. Destaca un leve aumento en la media de la $\mathrm{SaO}_{2}$ mínima (85,5\% [RIC: $82,0-88,3$ ] vs $86,0 \%$ [RIC: 84,0 $88,0] ; p=0,859$ ) y una tendencia a una disminución del índice de desaturaciones (10,2 eventos/hora [RIC: 2,8-13,3] vs 7,1 eventos/hora [RIC: 4,8-8,6]; $p=0,953)$. Respecto a las variables que evalúan la calidad de sueño se observó una tendencia a un aumento en la proporción de la etapa profunda de sueño NREM (N3) y a una disminución de microdespertares (Tabla 2). En la Figura 1 se grafican los principales resultados en las variables clínicas y polisomnográficas antes y después de la TMO. 
Tabla 2. Variables polisomnográficas de calidad de sueño antes y después de la TMO

\begin{tabular}{|lccc|}
\hline & $\begin{array}{c}\text { PSG PRE-TM0 } \\
\text { Mediana (p25-p75) }\end{array}$ & $\begin{array}{c}\text { PSG POST-TM0 } \\
\text { Mediana (p25-p75) }\end{array}$ & $\begin{array}{c}\text { Wilcoxon test } \\
\mathrm{p}>\mathrm{z}\end{array}$ \\
\hline Tiempo total de sueño (minutos) & $418,3(383,8-451,1)$ & $415,5(296,0-460,3)$ & 0,5303 \\
Eficiencia del sueño (\% del TST) & $80,1(76,8-86,5)$ & $91,8(79,7-95,0)$ & 0,7213 \\
Latencia inicio del sueño (minutos) & $12,0(9,4-35,3)$ & $12,8(5,5-17,9)$ & 0,5548 \\
Latencia inicio etapa REM (minutos) & $209,8(123,0-289,0)$ & $97,5(68,4-255,0)$ & 0,1394 \\
Etapa N3 (\% del TST) & $19,4(10,8-23,0)$ & $21,0(17,5-26,6)$ & 0,0997 \\
Etapa REM (\% del TST) & $7,5(4,5-12,2)$ & $8,0(4,7-15,6)$ & 0,9478 \\
Índice microdespertares (eventos/hora) & $13,0(11,6-22,2)$ & $12,6(12,2-15,7)$ & 0,0910 \\
\hline
\end{tabular}

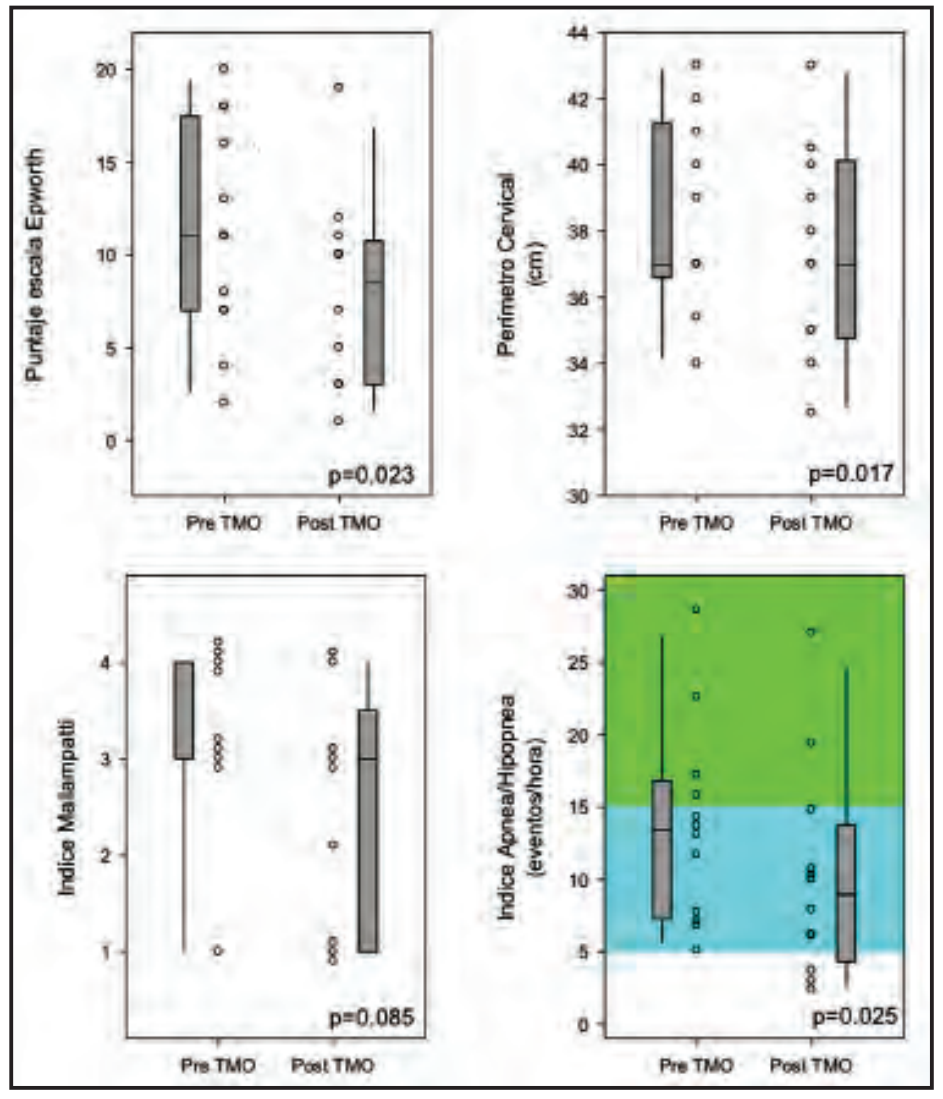

Figura 1. Los diagramas de caja y bigotes indican los estadísticos mediana, percentil 5, 25, 75 y 95 de la muestra antes (preTM0) y después (posTMO) de la terapia miofuncional orofaríngea. Cada sujeto se indica mediante círculos abiertos conectados mediante línea punteada. La significación estadística fue obtenida mediante el test de Wilcoxon para muestras pareadas. En el gráfico de índice de apnea/hipopnea se indica el nivel de severidad mediante código de colores: verde =SAHOS moderado, celeste =SAHOS leve. 


\section{DISCUSIÓN}

En nuestra muestra de 12 pacientes con SAHOS leve a moderado se observó una disminución en $32,8 \%$ de la mediana del IAH en la polisomnografía realizada entre tres a seis meses después de finalizada la TM0. Destacamos que el $41,6 \%$ de los pacientes redujo su categoría de severidad del SAHOS, con $25 \%$ de sujetos que alcanzaron un IAH normal. Dicho efecto se asocia a una disminución significativa del puntaje en la escala de somnolencia de Epworth y del perímetro cervical; además de una tendencia a disminuir el índice de Mallampatti. Estas variables clínicas se relacionan con un mayor riesgo de padecer SAHOS ${ }^{11}$. En la polisomnografía posterior a la TMO se observó una tendencia a una mejoría de la calidad de sueño evidenciada en un aumento en la etapa N3 y disminución del índice de microdespertares.

Particularmente relevante es el estudio randomizado y controlado de Guimaraes y cols ${ }^{12}$ realizado en una serie demográficamente comparable a la nuestra, que demuestra con TMO una respuesta clínica (disminución en 6 puntos en la escala Epworth), anatómica (disminución del perímetro cervical en $1,1 \mathrm{~cm}$ ) y funcional (reducción de IAH en 9,7 puntos). Estos resultados han sido confirmados en diversas series de pacientes con SAHOS, resumidos en un reciente metaanálisis ${ }^{13-15}$. Otras ventajas descritas en la respuesta a la TMO son la reducción en $30 \%$ a $51 \%$ la intensidad y frecuencia de los ronquidos ${ }^{16,17}$, y una mejor adherencia al uso de CPAP cuando se la indica como terapia coadyuvante ${ }^{18}$. Diaféria y cols demostraron que Ios pacientes con SAHOS tratados con TMO más CPAP tenían una mayor mejoría en los parámetros de calidad de vida respecto a aquellos pacientes tratados sólo con CPAP ${ }^{19}$.

Nuestros resultados fueron obtenidos en un contexto clínico real, con pacientes no seleccionados, donde todos los sujetos tenían más de 50 años de edad y eran portadores de enfermedades crónicas. No tenemos conocimiento de otras publicaciones en Chile que investiguen los efectos de la TMO en pacientes adultos con trastornos respiratorios del sueño. Nuestra principal dificultad para obtener un mayor número de casos fue contar con una polisomnografía de control, ya que es un examen poco accesible en el contexto de nuestro sistema público de salud. Sin embargo, cabe mencionar que en un reciente metaanálisis con un total de 9 estudios seleccionados que evaluaban la efectividad de la TMO en SAHOS, sólo 3 de ellos contaban con más de 20 casos de estudio ${ }^{10}$.

Durante el período en que los pacientes fueron derivados a su tratamiento con ejercicios miofuncionales, nuestro hospital no contaba con la posibilidad de indicarles terapia con dispositivo de avance mandibular (DAM). Al igual que la TMO, el DAM está indicado principalmente en pacientes con SAHOS leve a moderado. Los metaanálisis publicados describen una reducción del IAH entre $30 \%-72 \%$, una tasa de respuesta total (lograr IAH $<5$ eventos /hora) o parcial (reducción del IAH mayor al $50 \%$ del valor basal) en el $45 \%-100 \%$ de los pacientes. El DAM también disminuye el índice de ronquidos, el índice de microdespertares y mejora los índices de calidad de vida ${ }^{20-22}$. No encontramos estudios que comparen directamente la eficacia terapéutica de los diversos DAM y la TMO en las bases de datos revisadas en el intervalo 2015 y 2019 (www.ncbi.nlm.nih.gov/pubmed y scholar. google.cl).

El año 2006 Puhan y cols ${ }^{23}$ demostraron una disminución significativa del índice de eventos respiratorios durante el sueño en pacientes a quienes se les enseñó a tocar didgeridoo, un instrumento musical de viento que, para su correcta ejecución, requiere de un gran esfuerzo de estructuras musculares de la VAS. Esta experiencia dió pie al desarrollo posterior de la TMO en SAHOS. No se conoce completamente la fisiología implicada en el beneficio de los ejercicios miofuncionales: se atribuye a la disminución de la colapsabilidad de la VAS inducida por el entrenamiento muscular, en particular de una mejor función de musculatura dilatadora faríngea24. La TMO indicada a nuestros pacientes abarcaba una amplia gama de ejercicios isotónicos e isométricos para la mayoría de los músculos dilatadores de la faringe, aspecto que no permite establecer una relación entre el tipo específico de ejercicio y la respuesta terapéutica. Esta debilidad ha sido discutida en otros reportes ${ }^{9}$.

La aplicación de la TMO requiere ser aplicada por fonoaudiólogo integrado a un equipo de medicina del sueño y luego de una capacitación básica en esta terapia. No tuvimos complicaciones asociadas a los procedimientos. Por otra parte, tuvimos 
una alta tasa de abandono de las sesiones de TMO $(22,2 \%)$, algo que debemos evaluar y planificar para mejorar la adherencia al tratamiento. Sin embargo, no encontramos referencias a tasas de no adherencia en otras series. La evaluación miofuncional de la VAS a través de escalas estandarizadas también puede ayudar a distinguir a aquellos pacientes con alto riesgo de padecer SAHOS. Esto abre una ventana diagnóstica al considerar factores más dinámicos en la génesis de los trastornos respiratorios del sueño $0^{25,26}$. La alta prevalencia del SAHOS en la población adulta hace necesario que, junto a un aumento en la cobertura diagnóstica, exista un mayor espectro de alternativas terapéuticas al CPAP. Entre ellas debe considerarse la TMO, por ser efectiva y de bajo costo. Probablemente en el futuro todos los pacientes diagnosticados con

\section{BIBLIOGRAFÍA}

1. Ministerio de Salud, Gobierno de Chile. Encuesta Nacional de Salud ENS Chile 2009-2010. Capítulo 24, páginas 496-507; Disponible en: https://www.minsal.cl/portal/url/item/ bcb03d7bc28b64dfe040010165012d23.pdf. Consultado el 26 abril 2019.

2. Sullivan CE, Issa FG, Berthon-Jones M, Eves L. Reversal of obstructive sleep apnea by continuous positive airway pressure applied through the nares. Lancet 1981; 1: 862-5.

3. Rotenberg BW, Murariu D, Pang KP. Trends in CPAP adherence over twenty years of data collection: a flattened curve. J Otolaryngol Head Neck Surg 2016; 45: 43-51.

4. Baratta F, Pastori D, Bucci T, Fabianı M, Fabianı V, Brunori M, Loffredo L, Lillo R, Pannitteri G, Angelico F, Del Ben M. Long-term prediction of adherence to continuous positive air pressure therapy for the treatment of moderate/severe obstructive sleep apnea syndrome. Sleep Med 2018; 43: 66-70.

5. Cao MT, Sternibach JM, Guilleminault C. Continuous positive airway pressure therapy in obstructive sleep apnea: benefits and alternatives. Expert Rev Respir Med 2017; 11: 259-72.

6. Morgan TD. Novel Approaches to the Management of Sleep-Disordered Breathing. Sleep Med Clin 2016; 11: 173-87.
SAHOS deberán tratarse con TMO, independientemente de su severidad, ya que estudios recientes apuntan que también es una buena estrategia coterapéutica en casos de SAHOS severo. Se necesitan estudios a mayor escala para precisar la relación costo-beneficio de este tratamiento, así como estudios que comparen la efectividad de la TMO con otras terapias alternativas al CPAP como el DAM.

\section{CONCLUSIÓN}

En una población no seleccionada de pacientes con SAHOS leve a moderado, la terapia con ejercicios miofuncionales orofaríngeos reduce significativamente el IAH, el perímetro cervical y los puntajes de la escala de somnolencia de Epworth.

7. de Felício CM, da Silva Dias FV, Trawitzki LVV. Obstructive sleep apnea: focus on myofunctional therapy. Nat Sci Sleep 2018; 10: 271-86.

8. Kayamori F, BianchinI E. Effects of orofacial myofunctional therapy on the symptoms and physiological parameters of sleep breathing disorders in adults: a systematic review. Rev CEFAC 2017; 19: 868-78.

9. Kapur VK, Auckley DH, Chowdhuri S, Kuhlmann DC, Mehra R, Ramar K, Harrod CG. Clinical practice guideline for diagnostic testing for adult obstructive sleep apnea: an American Academy of Sleep Medicine clinical practice guideline. $J$ Clin Sleep Med 2017; 13: 479-504.

10. Kushida CA, Littner MR, Morgenthaler T, Alessi CA, Balley D, Coleman J JR, et al. Practice Parameters for the Indications for Polysomnography and Related Procedures: An Update for 2005. Sleep 2005; 28: 499-521.

11. Nuckton TJ, Glidden DV, Browner WS, Claman DM. Physical examination: Mallampati score as an independent predictor of obstructive sleep apnea. Sleep 2006; 29: 903-8.

12. Guimarães KC, Drager LF, Genta PR, Marcondes BF, LoRenZI-FILHo G. Effects of oropharyngeal exercises on patients with moderate obstructive sleep apnea syndrome. Am J Respir Crit Care Med 2009; 179: 962-6.

13. Сamacho M, Certal V, Abdullatif J, Zaghi S, Ruoff CM, Capasso R, Kushida CA. Myofunctional 
Therapy to Treat Obstructive Sleep Apnea: A Systematic Review and Meta-analysis. Sleep 2015; 38: 669-75.

14. Corrêa C de C, Berretin-Felix G. Myofunctional therapy applied to upper airway resistance syndrome: a case report. Codas 2015; 27: 604-9.

15. Rangel-León YJ, Rengifo-Varona ML, Gálvis-Gómez aM, Sarmiento-González MC, Giraldo-Cadavid LF. Rehabilitación de músculos orofaríngeos con ejercicios y electroterapia para el síndrome de apnea-hipoapnea obstructiva del sueño. Rehabilitación 2015; 49: 4-9.

16. Сamacho M, Guilleminault C, Wel JM, Song SA, Noller MW, Reckley LK, Fernandez-Salvador C, ZAGHI S. Oropharyngeal and tongue exercises (myofunctional therapy) for snoring: a systematic review and meta-analysis. Eur Arch Otorhinolaryngol 2018; 275: 849-55.

17. Leto V, Kayamori F, Montes MI, Hiralta RP, Gregório MG, Alencar AM, et AL. Effects of oropharyngeal exercises on snoring: a randomized trial. Chest 2015; 148: 683-91.

18. Diaféria G, Santos-Silva R, Truksinas E, Haddad FLM, Santos R, Bommarito S, Gregório LC, Tufik S, BITTEnCOURT L. Myofunctional therapy improves adherence to continuous positive airway pressure treatment. Sleep Breath 2017; 21: 387-95.

19. Diaféria G, Badke L, Santos-Silva R, Bommarito S, TufiK $S$, BitTencourt L. Effect of speech therapy as adjunct treatment to continuous positive airway pressure on the quality of life of patients with obstructive sleep apnea. Sleep Medicine 2013; 14: 628-35.
20. Basyuni S, Barabas M, Quinnell T. An update on mandibular advancement devices for the treatment of obstructive sleep apnoea hypopnoea syndrome. J Thorac Dis 2018; 10 Suppl 1: S48-S56.

21. Schwartz M, Acosta L, Hung YL, Padilla M, Enciso R. Effects of CPAP and mandibular advancement device treatment in obstructive sleep apnea patients: a systematic review and meta-analysis. Sleep Breath 2018; 22: 555-568.

22. Zhu Y, Long H, Jian F, Lin J, Zhu J, Gao M, Lal $W$. The effectiveness of oral appliances for obstructive sleep apnea syndrome: A metaanalysis. J Dent 2015; 43: 1394-402.

23. Puhan MA, Suarez A, Lo Cascio C, Zahn A, Heitz M, BRAendLI 0. Didgeridoo playing as alternative treatment for obstructive sleep apnea syndrome: randomized controlled trial. BMJ 2006; 332: 266-70.

24. Ferreira TS, Mangilli LD, Sassi FC, FortunatoTavares T, Limongi SC, Andrade CR. Speech and myofunctional exercise physiology: a critical review of the literature. J Soc Bras Fonoaudiol 2011; 23: 288-96.

25. Folha GA, Valera FC, de Felício CM. Validity and reliability of a protocol of orofacial myofunctional evaluation for patients with obstructive sleep apnea. Eur J Oral Sci 2015; 123: 165-72.

26. Felício CM, Lima MDRF, Medeiros APM, Ferreira JTL. Orofacial Myofunctional Evaluation Protocol for older people: validity, psychometric properties, and association with oral health and age. Codas 2017; 29: e20170042.

\footnotetext{
Correspondencia: Mario Díaz Sepúlveda Complejo Hospitalario San José, Servicio Salud Metropolitano Norte San José 1196, Independencia. Santiago, Chile E mail: diazmario1970@yahoo.com
} 